\title{
Fatigue Behaviour of Connectors used in Cable Harnessing through Cavity Formation Related Microstructural Degradation: A Failure Investigation Perspective
}

\author{
Mrityunjoy Hazra and Ashok Kumar Singh

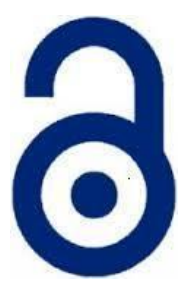 \\ Received: 23 February 2021 \\ Accepted: 16 August 2021 \\ Published: 01 January 2022 \\ Publisher: Deer Hill Publications \\ (c) 2022 The Author(s) \\ Creative Commons: CC BY 4.0
}

\begin{abstract}
Two separately failed electrical connector pieces during a vibration test were received for failure analysis. Chemical composition, hardness values and microstructures of the each of the connector material indicate that the material of construction is a die cast aluminium-silicon type of alloy, closely matching with the standard ANSI/AA B380 alloy. Intergranular and faceted fracture features are observed and failure mechanism is found to be fatigue dominated. The connectors failed by impact fatigue arising out of the loosening of the connector assembly. This has happened by cavity formation and/or growth related microstructural degradation processes. Initial casting pores as well as microstructural degradations such as interconnected pores have developed in service and their successive growth, decohesion and interconnection of each of primary Si particles and Al-Fe-Mn precipitates (along precipitate-matrix interface) have led the initiation of the crack under fatigue loading. Brittle as-cast microstructure (as typified by the precipitate-matrix interfacial cracking), existing vibratory loading and absence of any rise in temperature in the system have assisted the initial cavity (crack) formation and/or growth. Moreover, initial fitment related looseness is an additional factor in initiating and propagating this damaging mechanism.
\end{abstract}

Keywords: Connector, die cast aluminium alloy, failure analysis, impact fatigue, microstructural degradation, pores.

\section{INTRODUCTION}

Plated connectors meeting the quality assurance (QA) requirements for electrical performance and cleared by inspection agency are used for one system integration. The said connectors are employed for specific job of cable harnessing. The connectors are mated to each other and the whole system is subjected to vibration test. Two connector pieces have found to be damaged and/or broken after the completion of vibration test at room temperature. Present work describes the failure analysis of two such separately failed connectors.

\section{LITERATURE REVIEW}

\subsection{Cable Harnessing and Connector}

A cable harnessing system is an array of electrical cables or wires which transmit electrical power or mechanical/electrical signals [1]. It provides several advantages over loose wires and cables like: (i) better security against adverse effects of vibrations, abrasions, and moisture, (ii) optimum usage of space and (iii) reduced risk of a short. IPC's publication IPC/WHMA-A-620 for conforming and non-conforming requirements is consulted for this type of applications, unless it is customized by the user. For life-saving devices, military needs electronic products and for applications in harsh environments class 3 type of harnessing is required wherein its functioning is must for functioning of the whole assembly.

A connector is a device used to create electrical circuit and join electrical termination and it is one of the important components of a harness system. Connectors can be classified according to their functional operation and currentcarrying capacity into three groups: light, medium and heavy-duty connectors [2]. Light duty connectors are devices operating at voltages up to $250 \mathrm{~V}$ and carries current below 5A. A stable and low contact resistance and right connector material is essential for a successful operation of the connectors. Medium duty connectors operate at

Mrityunjoy Hazra $\bowtie$ and Ashok Kumar Singh

Defence Metallurgical Research Laboratory (DMRL)

P.O. Kanchanbagh, Hyderabad 500 058, India

E-mail: mhazra@dmrl.drdo.in

Reference: Hazra and Singh (2022). Fatigue Behaviour of Connectors Used in Cable Harnessing through Cavity Formation Related Microstructural Degradation: A Failure Investigation Perspective. International Journal of Engineering Materials and Manufacture, $7(1), 13-24$. 
voltages up to $1000 \mathrm{~V}$ and carries currents above 5A. Heavy duty connectors function at voltages up to hundreds of $\mathrm{kV}$ and carry currents up to tens of $k A$.

\subsection{Aluminium as Connector Material}

Aluminium is available in a variety of suitable alloys and forms which allow for the optimum choice for the manufacture of electrical connectors [3]. Aluminium alloys are defined by form, typical yield strength, minimum yield strength, elongation and percent of conductivity for specific applications. The alloys such as 6061-T6 in the extrusion form, 6063-T6 in the extrusion form, 356-T6 in the sand cast form, AXS 679 (380A) in the die cast form have been traditionally used as connector for various application types. Typical requirements of aluminium connectors for use with aluminium or copper conductors are: (a) adequate strength of the connector to prevent creep loss in the connection from exceeding the creep loss of the conductor, (b) strong enough clamping force (torque) to keep the connector operating temperature at a level below the operating temperature of the maximum size conductor and (c) high enough conductivity to provide adequate efficiency (minimum of $40 \%$ ).

Reliability of power connections is dictated by: (i) reliability requirements, (ii) basic feature of electrical contacts, (iii) connector design, (iv) degradation of electrical contacts, ( $v$ ) mitigating measures, ( $v i)$ new trends in electrical contact design and (vii) connector degradation. Connector degradation, as of now, constitutes one of the most important parts of determining the reliability. Degradation may have a highly deleterious effect on the operating cost of power network. Fig. 1 depicts various factors influencing the reliability of a connector performance.

\subsection{Degradation of Electrical Connectors and Contacts}

The damage of a connector proceeds relatively slowly and at a rate governed by the nature of a number of different processes operating along the contact zone and in the environment. This initial stage continues for a long time without causing any observable changes. However, when the contact resistance increases sufficiently to raise the local temperature, a self-triggering deterioration, resulting from the interaction of thermal, chemical, mechanical and electrical processes, would be in action and the contact resistance would rise again suddenly. Thus, there happens to be a synergistic effect between temperature rise and combined effect of interactions among thermal, chemical, mechanical and electrical effects, as soon as a certain temperature level is reached in the system [3, 4]. Hence, no deterioration would be noticed until the final stages of the connector life. Important degradation mechanisms are given in Fig. 2. Some of those are briefly described below.

\subsubsection{Creep}

It is a stress-, time- and temperature-dependent phenomenon and manifested by a dimensional change. Creep is an irreversible deformation offering the conductor termination by raising the potentials for overheating. Aluminium's tendency for creep is higher than those of the copper and many other conductors at normal operating as well as room temperatures. Additionally, aluminium's tendency to cold flow under pressure is significant. This leads to loosening of the terminals [2]. Creep coupled with cold flow is a factor challenging termination of aluminium conductors and connectors. For this reason, aluminium should have its own regulations and standards for torque settings of terminals, connection and terminating methods [5].

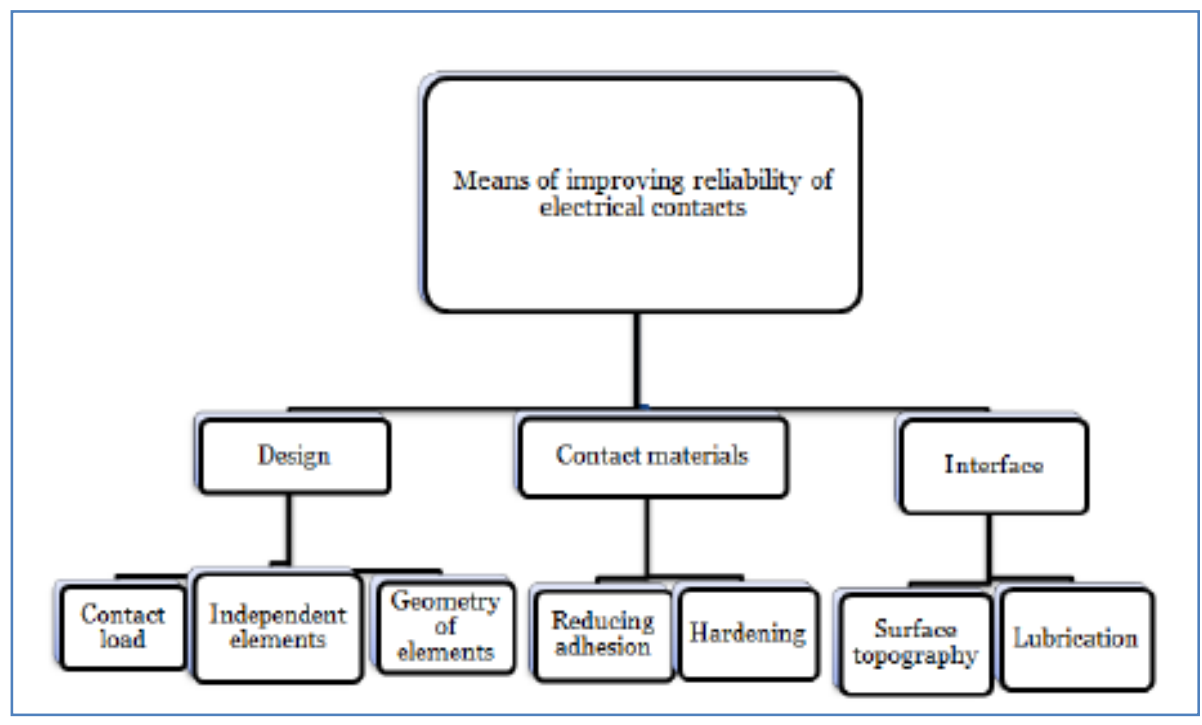

Figure 1: Factors influencing reliability of a connector assembly. 


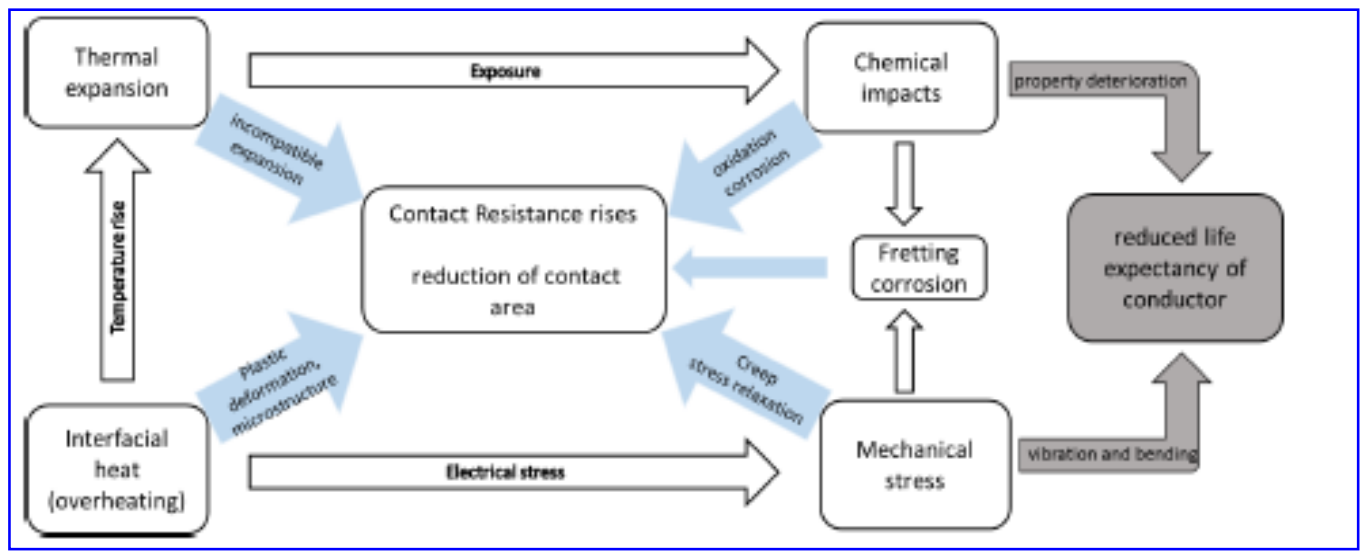

Figure 2: Schematic of degradation mechanisms in an aluminium connector assembly.

\subsubsection{Stress Relaxation}

It is also a stress-, time- and temperature-dependent phenomenon. However, it is manifested by a reduction in the contact pressure. It is higher for aluminium than those of the copper and other conductors. It occurs at high stress levels and is due to changes in metallurgical structure. It leads to reduction in contact pressure and consequently the increase in contact resistance. Sometimes, resistance is raised to the point of failure by short.

All metals are subjected to stress relaxation. Therefore, it is important to select the alloy carefully. There are three mechanisms by which alloys are strengthened: (a) solid solution strengthened, (b) dispersion-strengthened and (c) precipitation-hardened. The stress relaxation behaviour is related to the strengthening mechanism. Effect of cold deformation is nullified by time and temperature. Solid solution alloys have been found to be suitable for low contact forces. Higher contact forces in these alloys further require employment of cold deformation. Dispersion-strengthened alloys have good resistance to relaxation but are capable of offering only intermediate contact force. They are useful when low contact forces are required for use at elevated temperatures. Precipitation-strengthened alloys may be used for application requiring high contact force.

\subsubsection{Electroplasticity}

It is electricity induced enhanced plasticity event [6]. It increases creep / stress relaxation rates. Combined effect of Joule and electron-dislocation interaction makes the understanding of the governing mechanism behind electroplasticity quite complex. Thus, exact controlling mechanism behind a particular happening is mostly unknown. It has been shown by numerous studies that separation of the Joule heating and dislocation-electron interaction is possible. Studies related to the use of constant amplitude direct current during plastic deformation are often reported in this regard. Overall dislocation density has been found to decrease in this type of phenomenon.

\subsubsection{Oxidation and Corrosion}

Oxidation is accepted as the most serious degradation mechanism occurring in mechanical connectors. Corrosion starts at an exposed metal surface with the formation of a corrosion product and continues as long as reactants can diffuse through the product layer and sustain the reaction. Severity of the corrosion is determined by the composition and characteristics of the corrosion product layer. It may be caused by polluted atmospheres or by use of dissimilar metals in the contact. Oxide films and the products of corrosion on the contact surfaces reduce real contact area and increase contact resistance. The resultant increase in temperature further accelerates the rate of attack. On the other hand, formation of insulating $\mathrm{Al}_{2} \mathrm{O}_{3}$ is one of the most damaging factors, as it inhibits current flow, cause formation of hot spots in the contact spots. If contacts are designed to slide over each other (or wipe) when they are brought together or if connectors are frequently plugged and unplugged, the contact will have a self-cleaning action which helps to reduce contamination at the expense of increased wear. Contact lubricants are useful in this case, provided they do not dissolve any aggressive pollutant to increase its surface attack.

Aluminium conductors have higher tendency for corrosion because of its low zero potential. The galvanic corrosion occurs by connecting aluminium with other metals having higher zero potential. It weakens the conductor and leads to contact failure.

\subsubsection{Fretting}

Fretting is a surface damage occurring at the interface of contacting materials subjected to small oscillatory movements. It may occur at amplitude of less than $100 \mathrm{~nm}$. A report by Bock and Whitley [7] in 1974 was clearly convincing to the research community on the importance of fretting damage in electrical connections, although the possible degradation mechanism in telephone relays and switches were proposed around 1964 [8]. It is commonly manifested by overheating. There is a common lack of awareness on fretting phenomenon in electrical and/or 
electronic industries, as visibility and effect of wear debris and oxides would be appreciable after a significant residence time. Rapid increase in contact resistance and finally an open circuit is built up by accumulation of wear debris and oxides at contact zone between two connector surfaces over a time. Fretting may not directly lead to the failure, but it is certainly a damaging phenomenon. It is omnipresent by the fact that the oscillatory movements of the connectors are produced by mechanical vibrations, differential thermal expansion of connecting materials, relaxation of loads and also by heat produced at junction by switching on and off of the power.

\subsubsection{Thermal Expansion}

Longitudinal expansion is important since it often leads to slip in the joint followed by loosening. It is important that long bars are provided with a flexible element so that the movement can take place elsewhere. Aluminium has larger thermal expansion coefficient than the copper [2,4,5]. This may result in incompatible expansion between the terminal and a copper conductor. Coupling dissimilar metals with varying thermal expansion coefficients leads to loosening of the connection over time and increase contact resistance which in turn leads to arcing and overheating of the surface. This challenges the safety and reliability of the connection $[2,4,5]$.

\subsection{Connector Degradation, Loosening, Termination and Failure 2.4.1 Loosening}

Both the creep and cold flow lead to termination of connection by overheating (through raising the potentials) and loosening, respectively. Additionally, aluminium's tendency to cold flow under pressure is significant, again leading to loosening of the assembly. This creates chances of fatigue failure and voltage drop.

\subsubsection{Corrosion, Oxidation, Contact resistance, Temperature Increment and Contact Failure}

Oxide films and the products of corrosion on the contact surfaces reduce real contact area and increase contact resistance. This results in increase in temperature and further accelerates the rate of oxidation and corrosion attack. On the other hand, formation of insulating $\mathrm{Al}_{2} \mathrm{O}_{3}$ inhibits current flow, cause formation of hot spots in the contact spots. As mentioned above, the galvanic corrosion weakens the conductor and leads to contact failure.

\subsubsection{Thermal Expansion Coefficient}

Longitudinal expansion is important since it often leads to slip in the joint followed by loosening and increase contact resistance which in turn leads to arcing and overheating of the surface. This leads to contact failure.

\subsubsection{Issues with use of Dissimilar Metals at Termination [2-10]}

Using different metals at the termination join hampers handling high temperature, high vibration conditions and thermal shock due to aluminium's high thermal expansion coefficient, leading chances of failure.

The sequence of damaging phenomena beginning with entry of a connector assembly in service is described. A force is applied during termination and crimping which reduces the contact pressure resulting in non-gastight contact interface. At this point of time, corrosion and oxidation can occur even if the termination joint is sealed. This introduces loosening of the connection and increased contact resistance. It generates heat and further creep and cold flow of the conductor. Increased contact resistance leads to rise in temperature and therefore chance of aggravating oxidation, corrosion, creep etc. It is to be noted that all of damaging phenomena continue in a synergistic manner along with contact resistance. Additionally, increased resistance loosens the system and results into fatigue behaviour and high voltage drop.

\subsection{Feasible and Cost-effective Ways of Combating Connector Degradation}

Coating of the connection material is done for avoiding formation of insulating surface layer, mechanical wear and corrosion and promotes conductivity [10-14]. Use of nickel, tin, silver etc. have been traditionally employed for this purpose [11-14]. Aluminium, according to study done by Otsuka [10], for stabilization of contact resistance, requires a higher crimping compression than the copper and many other conductors in order to improve the wire retention force that enable electrical connection.

Use of lubrication - The conductive area is essential for a reliable electrical contact. This is affected by both internal and external factors. One of the most simple and efficient ways of achieving large contact points are by lubrication and mechanical abrasion. This together with application of a contact aid (grease) prevents oxidation of the metal.

\subsection{Prognostic Models for Contact Remaining Life}

Estimation of life time of a connector can be done by prognostic model where data collection of the component's performance from initiation to the final stage is an important prerequisite. In case of remaining life assessment of a connector, the contact interface is assumed to be homogenous with circular shaped $\alpha$-spots [16]. Additionally, it is assumed that the oxygen intrusion and growth of oxide film is the main factor affecting conductivity of the surfaces. 


\section{EXPERIMENTAL PROCEDURE}

The connectors were, at first, examined visually by naked eye and under magnifying glass. Photographs in the asreceived condition were taken and preserved for future reference, during the course of analysis. The failed parts were then sectioned from the main components for its further analysis. Later, those parts were put under forced air using air-blower to remove the loosely adhered particles and then examined under stereo microscope as well as scanning electron microscope (SEM). Fracture surface was cleaned ultrasonically in acetone after examination on probable surface corrosion debris and/or contamination, before fractographic study. After that, representative cross-sectional sample extraction from failed parts was carried out from near to the failed surface as well as from away from the failure location for detailed metallographic (optical microscopic and scanning electron microscopic) study. Samples along both longitudinal as well as transverse directions were extracted and microstructural features were studied in both un-etched and etched conditions. Bulk compositional analysis of the connector material was carried out by inductively coupled plasma-optical emission spectroscopy (ICP-OES) type wet chemical analysis technique. Dry combustion technique was used for hydrogen evaluation. Compositional analyses on each of the connectors were carried out with electron dispersive spectroscopy (EDS) attachment of the SEM. Samples were etched with Keller's reagent. Vickers micro hardness readings at 500gf were taken on metallographically prepared samples to correlate those with the respective microstructures so as to obtain a reasonably complete idea about the material.

\section{RESULTS}

\subsection{Visual Examination}

Figs. 3-5 show photographs of the damaged components in as-received condition. Fig. 3 exhibits one single received metal piece from a connector, named as connector 1 henceforth. As-received photographs of connectors 1 and 2 are displayed in Figs. 4 and 5, respectively. A dull greyish coating (possibly of cadmium) covers the surfaces of both the connectors. Fibrous type of fracture appearance seems to prevail on failed surfaces of both the connector 1 (Figs. 3 and 4) and connector 2 (Fig. 5). Ample of rub marks are noticed on surfaces near to and away from the failed surfaces.

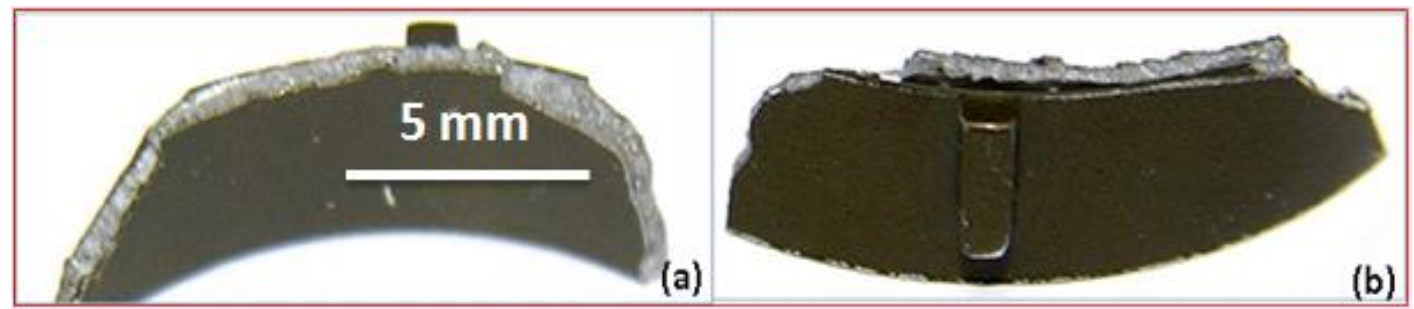

Figure 3: Photographs of the as-received fractured metal piece of connector 1 in different views

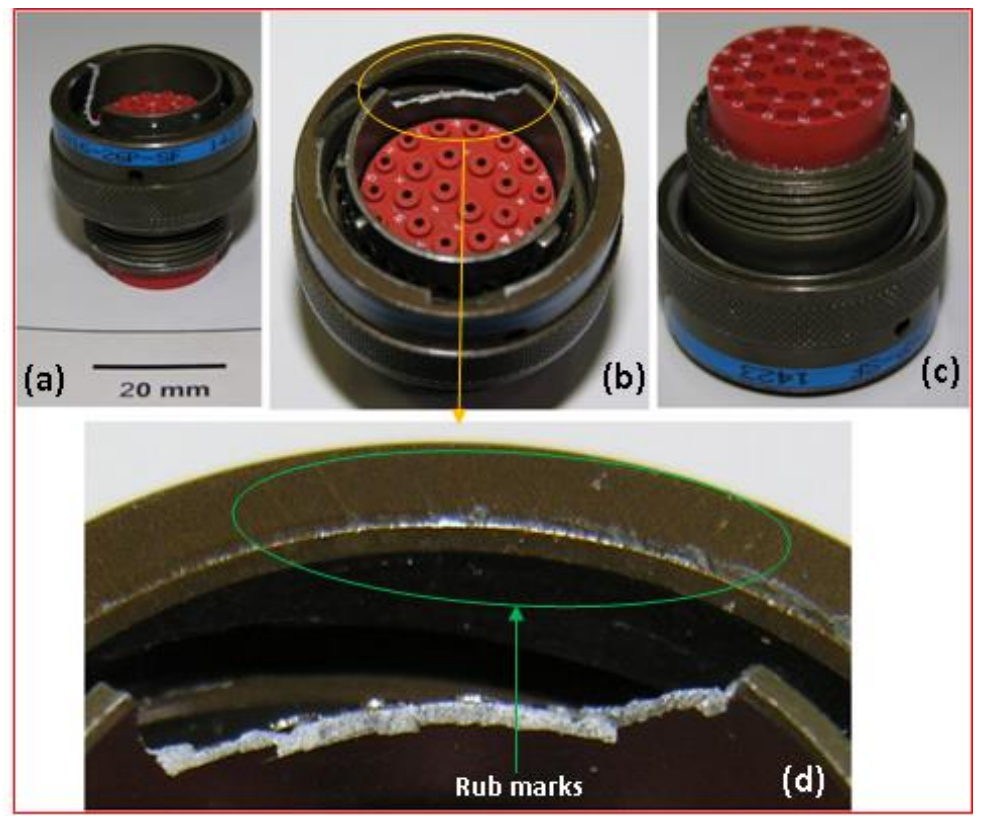

Figure 4: Photographs of the as-received connector 1 in different views 


\subsection{Fractography}

Figs. 6 and 7 show failed connectors and the metal piece as observed under stereo microscope. Only the gross fibrous fracture appearance is visible in this stage (Fig. 6). Significant rubbing has been observed on the whole connector surfaces for both the connectors.
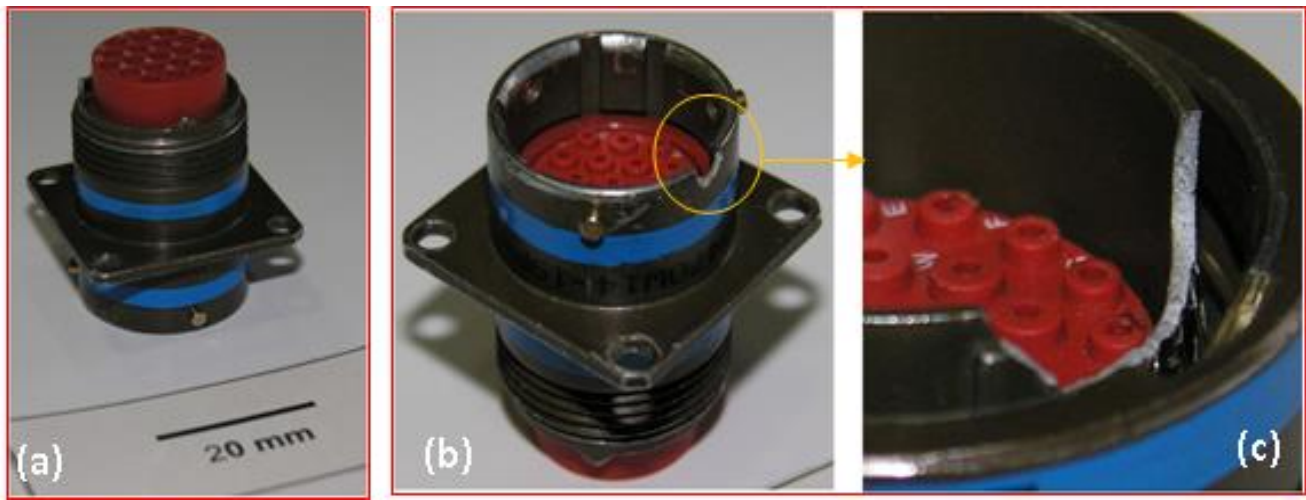

Figure 5: Photographs of the as-received connector 2 in different views

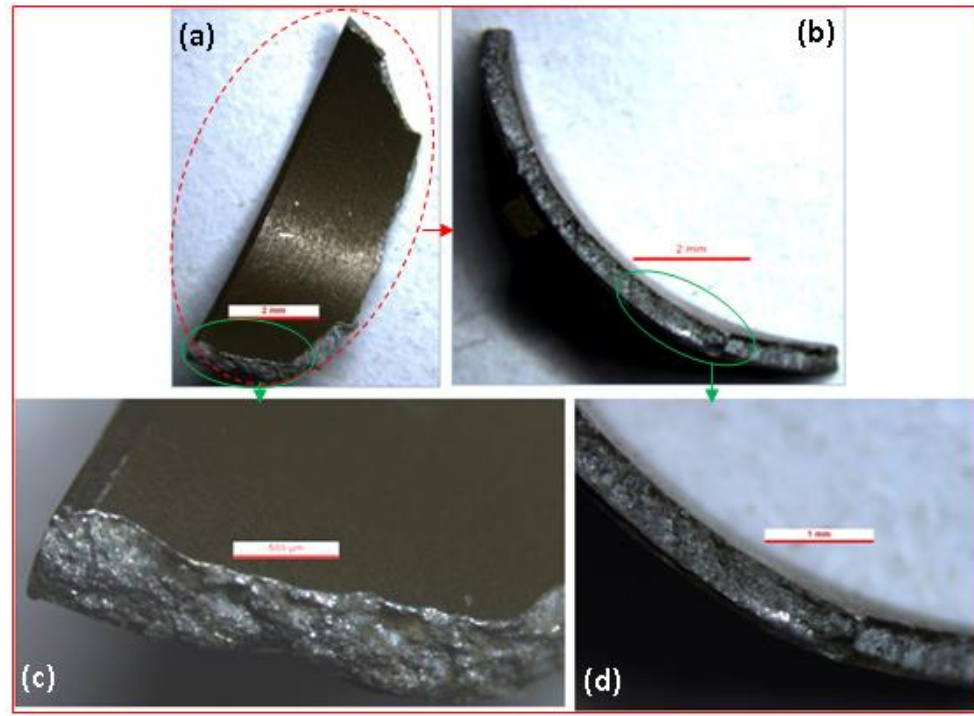

Figure 6: The as-received fractured metal piece of connector 1 in different views, as seen under stereo microscope

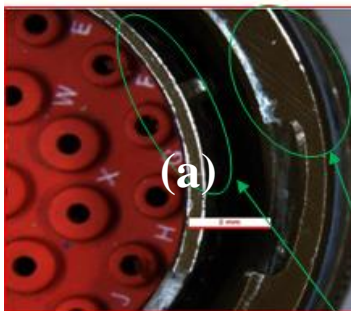

(a)
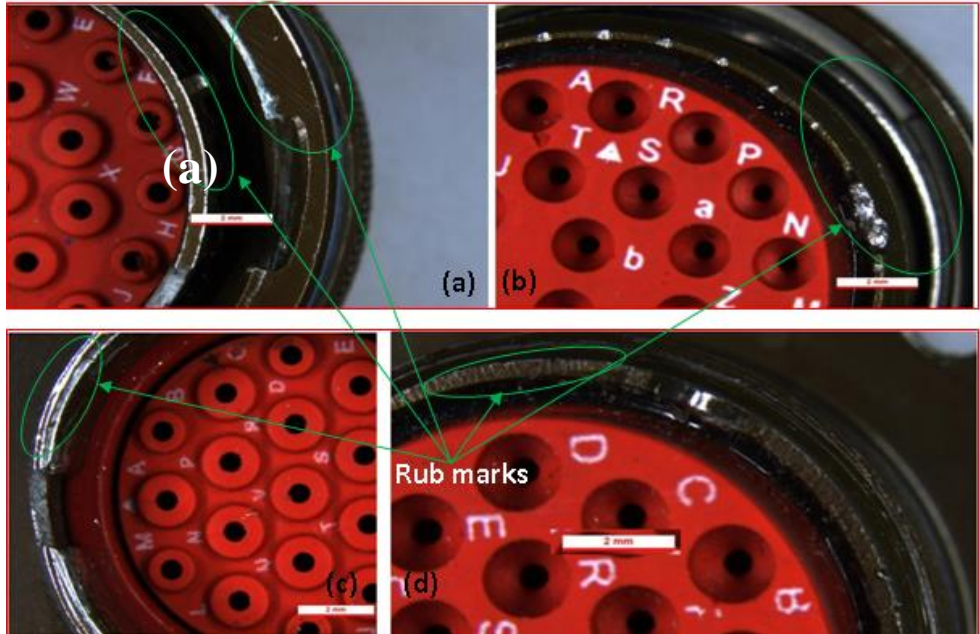

Figure 7: As-received connectors in different views, as seen under stereo microscope. (a, b) connector 1, (c, d) connector 2 . 
Figs. 8-10 exhibit failed connectors and the metal piece as observed under SEM. Intergranular and faceted fracture features have been observed predominantly. Indication of fatigue failure mechanism is clearly revealed in each of the fractographic images. Secondary cracks with moderate frequency were noticed on fracture surfaces of both the connectors. Significant rub marks have also been noticed here just adjacent to the fracture surfaces (Figs. 8 and 10).

\subsection{Microstructure}

Fig. 11 shows longitudinal and transverse metallographic sections of connector 1 in un-etched condition at various magnifications. The same for connector 2 has been presented in Fig. 12. Significant amount of casting porosity is seen in microstructure of both the connectors. Moreover, few precipitates with appearance of fragmented particles are also noticed.
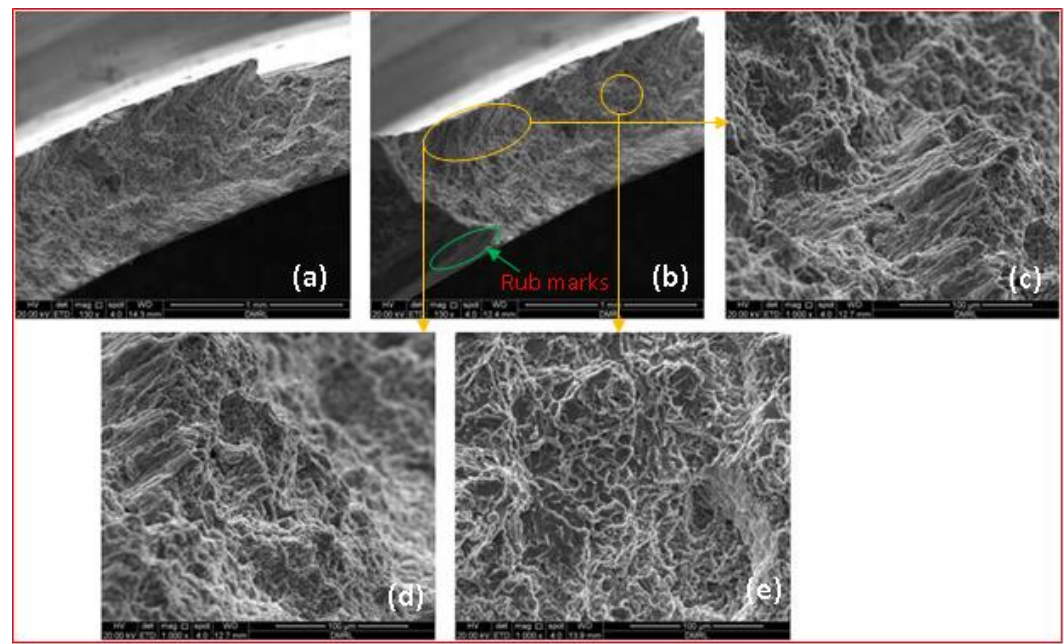

Figure 8: Fracture surface of dislodged metal piece of connector 1 in different views, as seen under SEM
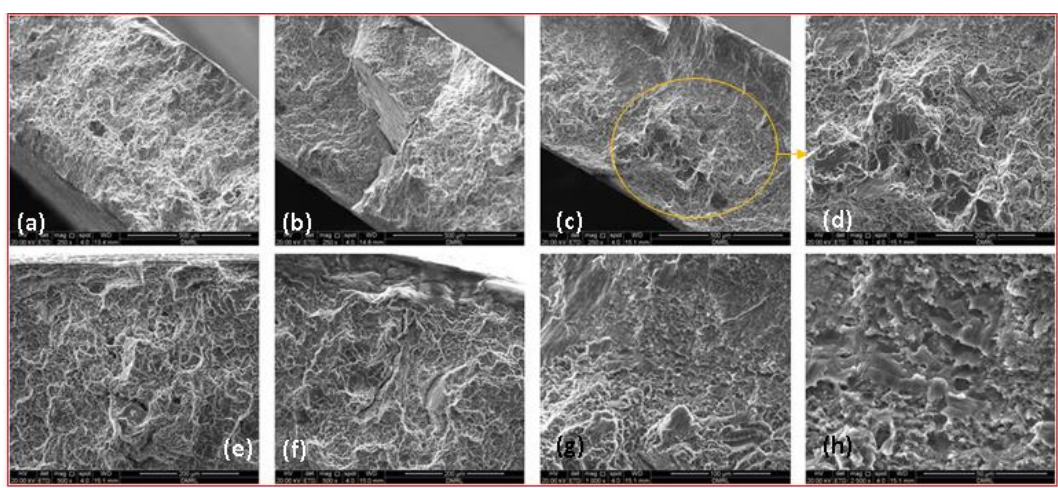

Figure 9: Fracture surface of mating part of connector 1 in different views, as seen under SEM
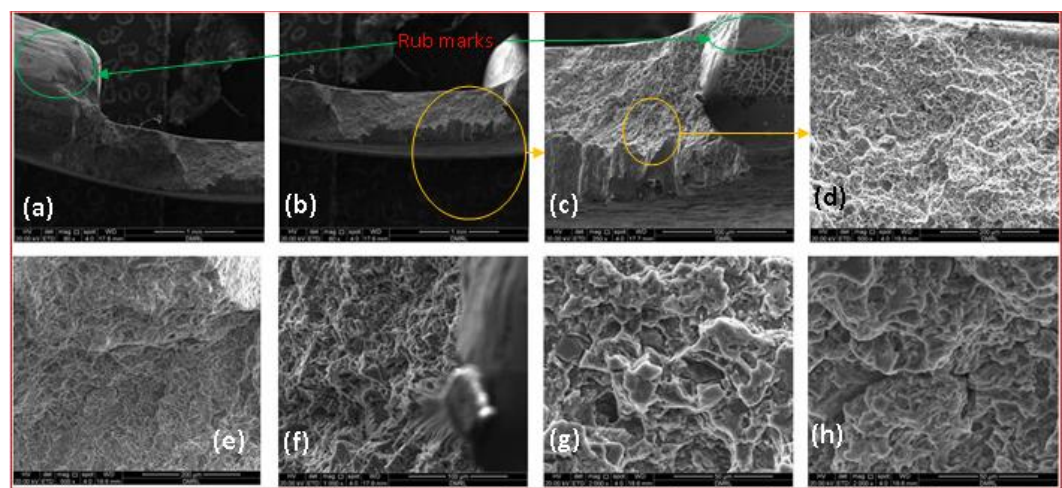

Figure 10: Fracture surface of connector 2 in different views, as seen under SEM 
The etched microstructure reveals typical heterogeneous cast appearance, consisting of dendrites and equiaxed grains (Fig. 13). SEM analysis displays well developed eutectic of Al-Si (phase A), as shown in Figs. 14 and 15. Al-Cu rich phases (marked B) are observed at few peripheral and nodal regions of these eutectics. On the other hand, in some of the regions, the Al-Fe-Mn rich phases (phase F) are found within the Al-Si eutectics. Primary Si particles of varying sizes and shapes (phase D) are observed within Al-Si rich matrix throughout the microstructure. Besides, Al-Fe rich rod shaped phases (phase $G$ ) are found to be spreaded across the eutectic and matrix regions. Occasional presence of W-Fe rich foreign phases is observed. Various types of cavities are seen, viz. (a) inherent casting pores of two types (marked $\mathrm{E}_{\text {and }} \mathrm{E}^{\prime}$ ), (b) cavity formed by fracture of primary Si particles and its interconnection (marked $\mathrm{D}^{\prime}$ ), (c) those formed by fracture of Al-Fe-Mn precipitates and its interconnection (marked $\mathrm{F}^{\prime}$ and $\mathrm{F}^{\prime /}$ ). Fractured, dislodged and interconnected $\mathrm{Al}-\mathrm{Fe}-\mathrm{Mn}$ precipitates appear as grain boundary cracks (marked $\mathrm{F} /$ ). On the other hand, some inherent casting cracks and/or interconnected inherent pores (marked $\mathrm{E}^{\prime}$ ) become visible as curvy line. A coating of average thickness $5 \mu \mathrm{m}$ is observed on each of the connector surfaces, as represented by the SEM image displayed in Fig. 16. EDS analysis on coating reveals that it is of Ni-P type. Various types of phases and cavities are enlisted in Table 1.
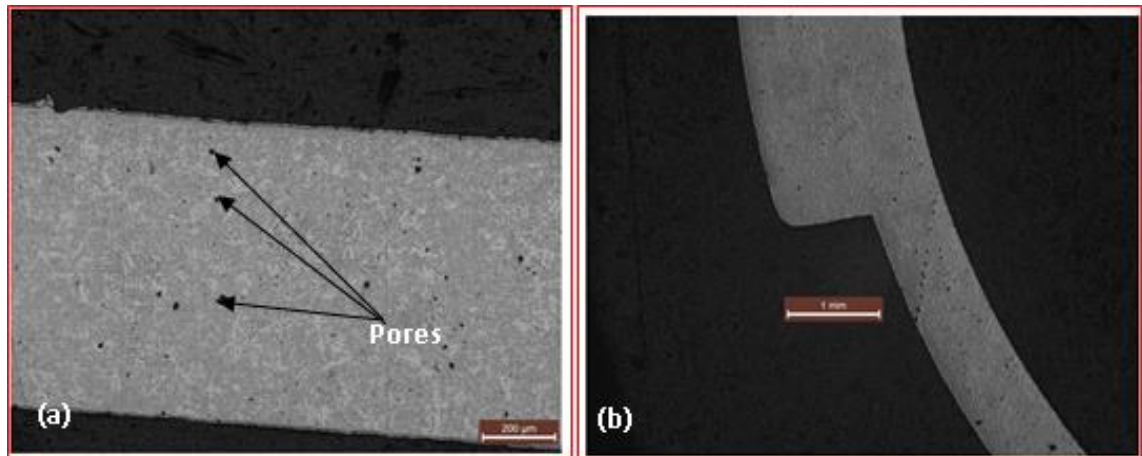

Figure 11: Optical microstructure of connector 1 for longitudinal (a) and transverse (b) sections in different magnifications. Un-etched.
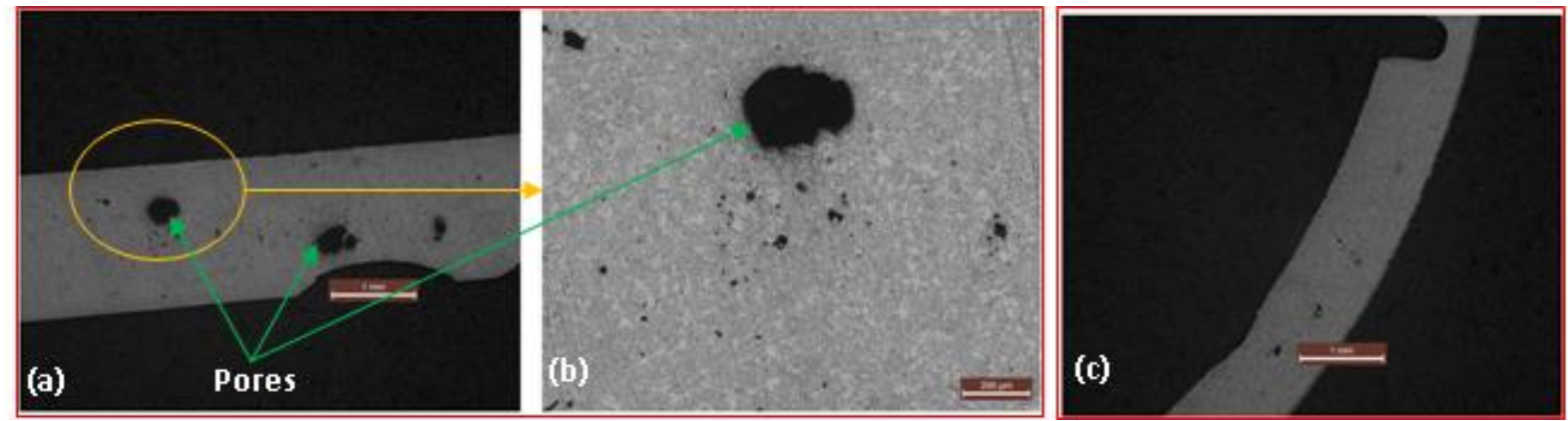

Figure 12: Optical microstructure of connector 2 for longitudinal $(a, b)$ and transverse 1 sections in different magnifications. Un-etched.
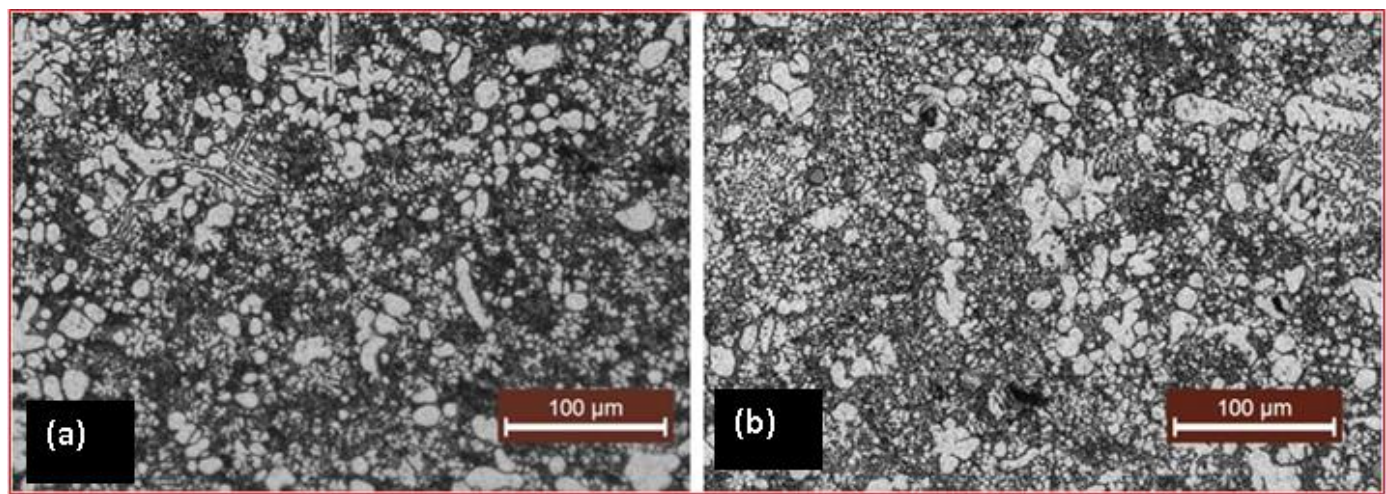

Figure 13: Representative optical microstructure of connectors (connector 1 here) for longitudinal (a) and transverse sections (b). Etchant: Keller's reagent. 


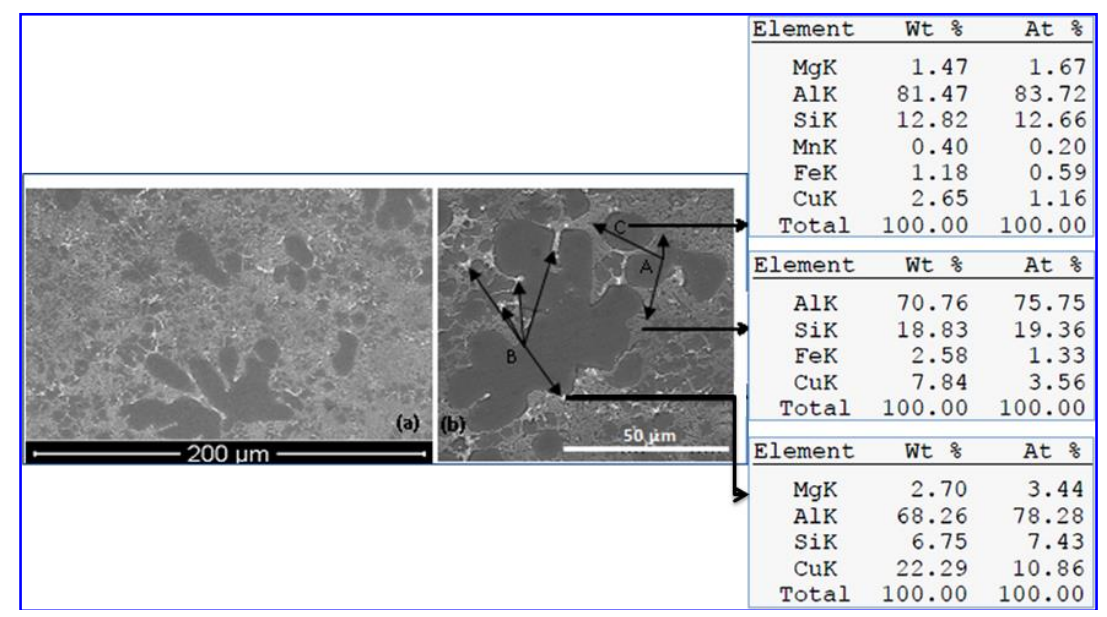

Figure 14: Representative SEM microstructure of longitudinal section of connector 1 in different magnifications (a,b). Etchant: Keller's reagent.

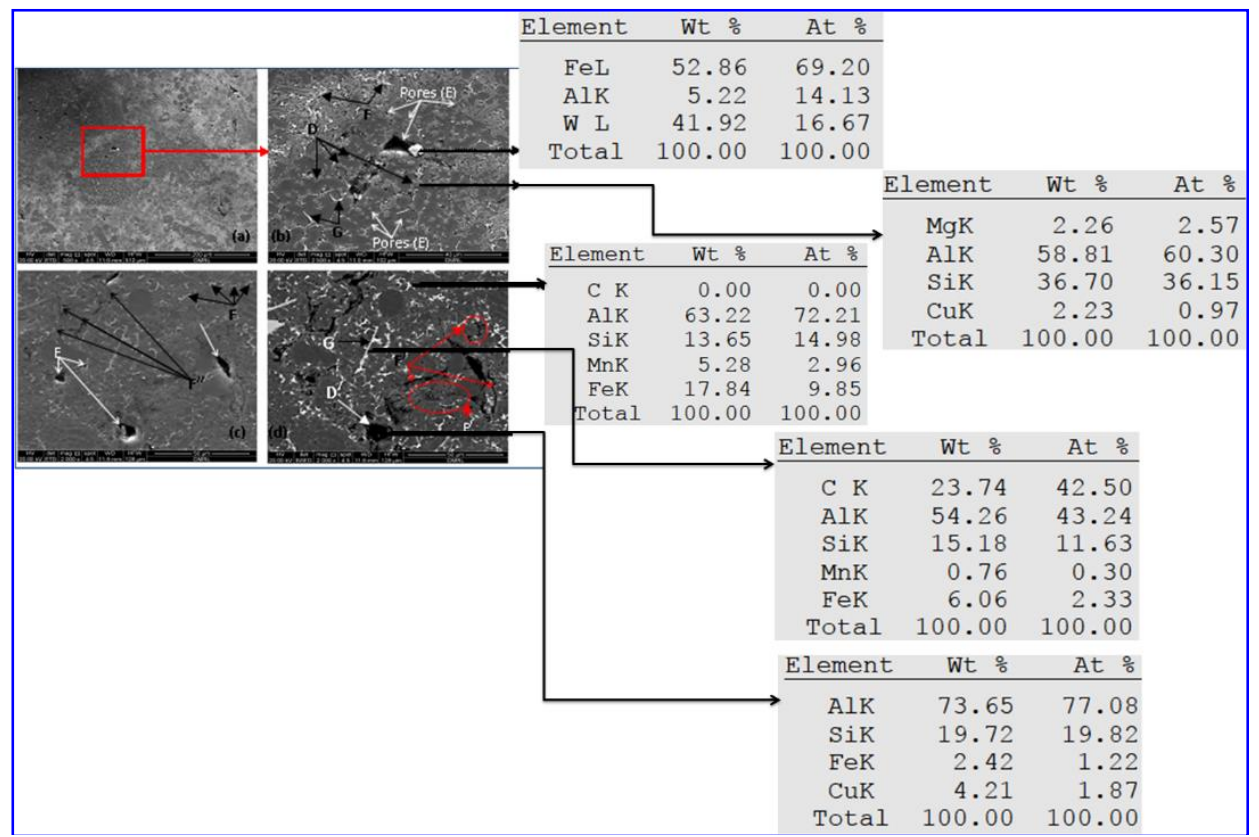

Figure 15: Representative SEM microstructure of connectors - $(a, b)$ transverse section of connector 1, longitudinal section of connector 2 in SE I and BSE (d) modes, Etchant: Keller's reagent.

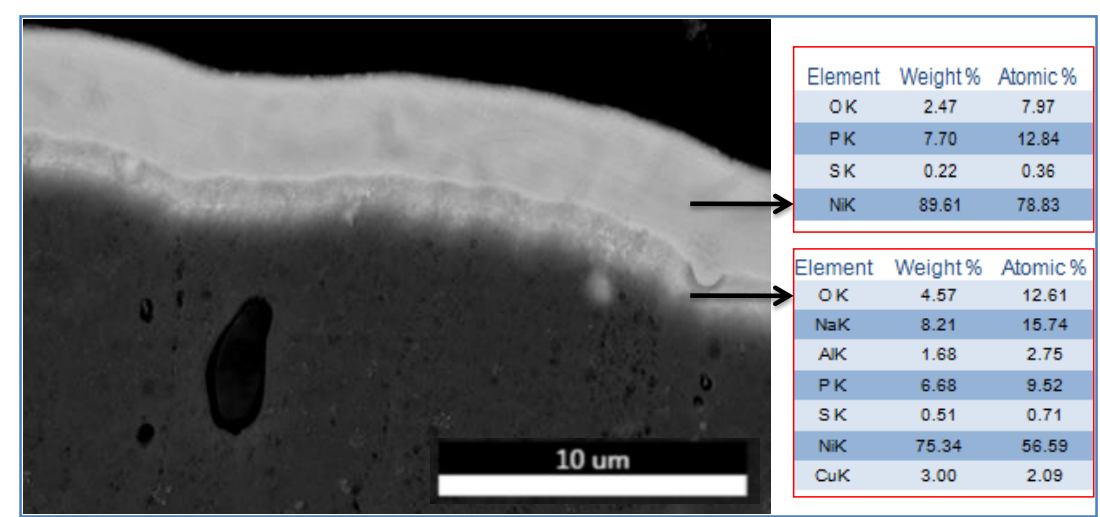

Figure 16: One representative SEM image showing coating on the connectors along with corresponding EDS analysis, Etchant: Keller's reagent. 
Table 1: List for various phases and cavities

\begin{tabular}{cl}
\hline Marked phases in Figs. 15 and 16 & Description \\
\hline A & Al-Si eutectic \\
B & Al-Cu rich phases \\
C & Matrix \\
D & Primary Si particles \\
F & Al-Fe-Mn rich phases \\
C & Al-Ferich rod shaped phases \\
D' & Cavities formed by fracture of primary Si particles \\
E & Inherent casting pores, type I \\
E' $^{\prime}$ & Inherent casting pores, type II \\
F $^{\prime}$ & Cavities formed by fractured and dislodged Al-Fe-Mn precipitates \\
F $^{\prime \prime}$ & Cavities formed by interconnection of cavity type F' \\
\end{tabular}

Table 2: Chemical composition of failed connector materials

\begin{tabular}{cccccccccccc}
\hline & \multicolumn{10}{c}{ Content (Wt. \%) } \\
\hline Material & $\mathrm{Cu}$ & $\mathrm{Si}$ & $\mathrm{Zn}$ & $\mathrm{Fe}$ & $\mathrm{Mn}$ & $\mathrm{Ni}$ & $\mathrm{Mg}$ & $\mathrm{Sn}$ & $\mathrm{Al}$ & $\mathrm{H}$ \\
Connector 1 & 2.69 & 6.97 & 0.47 & 0.77 & 0.33 & 0.089 & 0.055 & 0.023 & $\mathrm{Rem}$ & 0.0030 \\
Connector 2 & 3.39 & 9.98 & 1.40 & 1.10 & 0.29 & 1.07 & 0.13 & 0.023 & $\mathrm{Rem}$ & 0.0024 \\
\hline
\end{tabular}

\subsection{Chemical Composition and Hardness}

Table 2 reveals bulk compositions and hydrogen content of two failed connector material. Vickers hardness value taken at $500 \mathrm{gf}$ load for the connector materials (connector 1 and connector 2) is found to be $\sim 90 \pm 03 \mathrm{HV}$.

\section{DISCUSSIONS}

\subsection{Material of Construction for the Connectors: Identity and Suitability}

Chemical composition, hardness value and microstructure of the connector material indicate that the material of construction of the connectors is a die cast Al-Si type of alloy, closely matches with the standard ANSI/AA B380 alloy $[17,18]$. Coating of $\mathrm{Ni}-\mathrm{P}$ type of around $5 \mu \mathrm{m}$ thickness is observed on the connector surfaces, while standard practice is to have Ni coating of $20 \mu \mathrm{m}$ thickness ( $\mathrm{min}$ ) followed by a $10 \mu \mathrm{m} \mathrm{Ag}$ coating on top for lowering contact resistance arising out of aluminium oxides $[19,20]$. Porosity is observed to be distributed quite uniformly throughout the microstructure. Die cast aluminium alloys of this type has been traditionally recommended and popularly used in electronic connectors and housings, because of their excellent electrical performance and shielding properties. Usually recommended and most popular aluminium alloy for electrical and/or connector is $A 380[17,18]$. B380 is a sub-class of $\mathrm{A} 380$ with restricted $\mathrm{Zn}$ content $(\approx 1 \mathrm{wt} . \%)$ as compared to that $(\approx 3 \mathrm{wt} . \%)$ in $\mathrm{A} 380$ alloy [18]. Use of B380 probably suits better than the $\mathrm{A} 380$ in the present type of applications as the former has higher high temperature capability and lower susceptibility for hot tearing.

Different casting processes can lead to different amounts of porosity (both in terms of shrinkages and gaseous porosities) due to their specific peculiarities [18,21]. Die cast products, especially high pressure die cast (HPDC) alloys usually contain higher amount of porosity than that is found in conventional and also in advanced casting processes. However, in absence of the intended specification of the material for connector type of applications, it is not possible to comment on the desirable level of porosities in the presently investigated material system. However, it should be noted that porosity affects fatigue properties of cast aluminium alloy very significantly. There are different sources of porosity and defects in aluminium casting [21]. These are (i) alloy related and/or (ii) process-related. Alloy related issue concerns with the volume reduction associated to the liquid-solid transformation, which may result in macroporosities (due to the presence of hot spots) as well as in microporosities (interdendritic shrinkages). Process related issue include (a) evolution of hydrogen and its entrapment within the material structure, due to strong decrease in its solubility during liquid to solid phase transformation of the material, (b) entrapment of gases (and of derived oxides) due to excessive turbulence in flow of molten alloy during the filling stage. Combined effects of alloy and process related issues include: (a) establishment of stress states during solidification, causing cracks and hot tears into the cast alloy and (b) presence of inclusions and the development of gases from cores.

\subsection{Failure Mechanism}

Intergranular and faceted fracture features are observed. Fracture surfaces of both the connectors have revealed evidence of fatigue dominated mechanism. The probable initiation regions of fatigue failure seem to be the cavities present in the microstructure [22,23]. Cavities are present in various forms, viz. original casting pores, interconnected pores in service, cracking and decohesion of various microstructural precipitates. Presence of ample of rubbed zones on connector surface at region close to the fracture as well as at regions away from the fracture (Figs. 4,7,8 and 9) indicates continuous hitting between two mating connectors. This is due to initial fitment related looseness and/or by loosening of the system due to cavity formation related degradation processes of the connector material [22]. 
However, based on background information received from the user, the fitment related looseness has a remote chance of occurrence. Interestingly, in the present case, again by background information, chance of creep type of high temperature degradation is nil, as vibration tests are conducted at room temperature. Also, there is no increase in temperature of the system as a result of increase in contact resistance by oxide formation and/or relaxation of the system over a time etc., as monitored continuously. Thus, the above mentioned cavity formations and their interconnections are aided only by vibratory type of loading. The said repeated hitting of mating connector surfaces for presently used connector like cast materials with very low ductility $(\approx 3 \%$ elongation value) resembles almost to phenomenon like repeated impact loading $[17,18]$. This indicates the presence of rapid rate of loading in cyclic (fatigue) mode.

Now, as is mentioned earlier, material weakness in form of various types of cavities (Table 1) is observed in the microstructure. This weakness often appears like grain boundary cracking. Few other areas are full of regions with decohesion of Al-Fe-Mn precipitates. Also, occasional contribution of unexpected phases containing Fe and impurities like $W$ (and expected to be brittle one) to the cracking phenomenon observed. This type of microstructure justifies very much the granular and/or layered fracture appearance of the failed connectors [22].

The fatigue type of loading evolved from connectors has in fact, aided enlargement and/or interconnection and/or alignment of the already present cavities along a particular direction. Also, it aided the decohesion of Al-Fe$\mathrm{Mn}$ precipitates. Thus, the fatigue type of loading had substantial contribution to the type of fracture observed, along with original microstructural features (defects). On the other hand, low ductility of the material and thus its exposure to rapid rate of loading (under continuous hitting and rubbing of mating surfaces) has accelerated the failure process by accelerating the formation of crack of sufficient length for inducing high propagation rate by quick interconnection of cavities. Simultaneous actions of fatigue as well as rapid rate of loading have produced intergranular and/or layered fracture appearance of an already defected microstructure without dimples.

\section{CONCLUSIONS}

This research investigates the fatigue failure perspective of connectors used in cable harnessing. Based on this research the following conclusions were drawn.

1. The connectors have failed by fatigue arising out of the loosening of the connector assembly due to cavity formation related microstructural degradation processes.

2. Initial casting pores and microstructural degradations such as interconnected pores developed in service, successive cracking, decohesion and interconnection of each of primary Si particles and Al-Fe-Mn precipitates have led the initiation of the crack under fatigue loading.

3. Fatigue type was found to be of impact type.

\section{ACKNOWLEDGEMENT}

The authors would like to thank Dr. G Madhusudhan Reddy, Outstanding Scientist and the Director, DMRL for his constant encouragement to work on the present field. Also, funding from DRDO is gratefully acknowledged.

\section{REFERENCES}

1. Www.cableharnessing.co.uk, last accessed on 27.02.2020.

2. Braunovic, M. "Power Connectors," in Electrical Contacts Principles and Applications, 2 ed., P. G. Slade, Ed., CRC Press Taylor \& Francis Group, 2014, 233-366.

3. Hamedi, E. Electrical connection for aluminium conductors in automotive applications: Prestudy of available solutions for electrical connection methods of aluminium cables, Inom Examensarbete Material Design, Avancerad Nivå, 30 HP, Stockholms Verige 2017.

4. Chapman, D. Copper in Electrical Contacts, Copper Development Association Publication No 223, European Copper Institute Publication No Cu0169, July 2015.

5. SS-ISO 6722-2. Road vehicles-60V and 600V single-core cables-part 2: dimensions, test methods and requirements for aluminium conductor cables.

6. Tiwari, J., Pratheesh, P., Bembalge, O.B., Krishnaswamy, H., Amirthalingam, M., Panigrahi, S.K., Microstructure dependent electroplastic effect in AA 6063 alloy and its nanocomposites, Journal of Materials Research and Technology, 2021, 12, pp. 2185-2204.

7. Bock, E.M., Whitley, J.H., "Fretting corrosion in electrical contacts", Electrical Contacts, 1974, Illionis Institute of Technology, Chicago, 1974, pp. 128.

8. Braunovic, M., Fretting Damage in Tin-Plated Aluminium and Copper Connectors, IEEE Transactions on Components Hybrids and Manufacturing Technology 12(2):215 - 223, 1989, doi:10.1109/33.31426.

9. Wert, C.A., Thomson, R.M. Physics of Solids, 2nd edition, McGraw-Hill Book Company, New York, 1970.

10. Otsuka, T., Hirai, H., Ono, J. "Crimping Technology of Alumium Wire for Automotive Wire Harness," Auto Networks Technologies Ltd, Tokyo, 2012.

11. Yasuda, K., Umemura, S., Aoki, T. "Degradation Mechanisms in Tin- and Gold-Plated Connector Contacts," IEEE Transactions on Components, Hybrids, and Manufacturing Technology, 1987, 10(3), 456-462. 
12. Rolin, T. D. et al. Salt Spray Test to Determine Galvanic Corrosion Levels of Electroless Nickel Connectors Mounted on an Aluminium Bracket. 2014.

13. Fujiwara, H. A patent for manufacturing methods to produce automotive power cables with Ni-layer.U.S. Patent No. 20030111256. 2003.

14. Cheerful, E. A method and compound for contacting an aluminium cable with a metallic,tinned contact clamp. D.E.Patent No. 10223397A12004.

15. Vogt, S., Thalmeier, M., Rill, D., Kindersberger, J. "High voltage aluminium connector (LEIKO)," München, Germany, 2013.

16. Braunovic, M., Izmailov, V., Novoselova, M. "A model for life time evaluation of closed electrical contacts," in Proceedings of the Fifty-First IEEE Holm Conference on Electrical Contacts, 2005, DOI: 10.1109/HOLM.2005.1518247.

17. Alloy Data: Aluminium Die Casting Alloys in Die Casting Aluminium Selection Guide, pp. A-3-1-97-A-3-3-97, published by NADCA.

18. Wang, L., Makhlouf, M., Apelian, D. Aluminium die casting alloys: alloy composition, microstructure, and properties-performance relationships, International Materials Reviews 1995, 40(06), 221-238.

19. Hamedi, E. Electrical connection for aluminium conductors in automotive applications Prestudy of available solutions for electrical connection methods of aluminium cables, Inom Examensarbete Materialdesign, Avancerad Nivå, 30 HP, Stockholms Verige 2017.

20. Yu, M. Aluminium cables in automotive applications, Prestudy of aluminium cable uses in scania products \& failure analysis and evaluation, Examensarbete Inom Materialteknik, Avancerad Nivå, 30 HP Stockholm Sverige 2016.

21. TALAT Lecture 1254: Fatigue in Al casting alloys: metallurgical aspect, by F. Bonollo and R. Tovo, European Aluminium Association, 1999.

22. Jaglinski, T., Lakes, R. Creep Behavior of Al-Si Die-Cast Alloys, Journal of Engineering Materials and Technology, Transactions of the ASME, 2004, 126, 378-383.

23. Zamani, M., Seifeddine, S., Anders E.W. J. Effects of microstructure and defects on tensile and fracture behaviour of a HPDC component: potential properties and actual outcome of an AC-44300 alloy, Light Metals, TMS (The Minerals, Metals \& Materials Society), 2014, 1077-1083. 